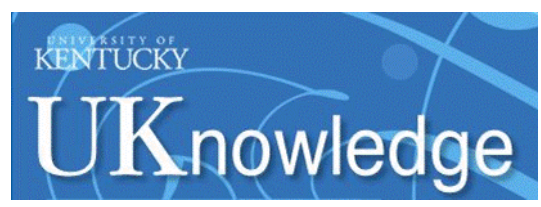

University of Kentucky

UKnowledge

$3-27-2018$

\title{
Intermediate-Line Emission in AGNs: The Effect of Prescription of the Gas Density
}

\author{
T. P. Adhikari \\ Polish Academy of Sciences, Poland \\ K. Hryniewicz \\ Polish Academy of Sciences, Poland \\ A. Różańska \\ Polish Academy of Sciences, Poland \\ B. Czerny \\ Polish Academy of Sciences, Poland \\ Gary J. Ferland \\ University of Kentucky, gary@uky.edu
}

Follow this and additional works at: https://uknowledge.uky.edu/physastron_facpub

Part of the Astrophysics and Astronomy Commons

Right click to open a feedback form in a new tab to let us know how this document benefits you.

\section{Repository Citation}

Adhikari, T. P.; Hryniewicz, K.; Różańska, A.; Czerny, B.; and Ferland, Gary J., "Intermediate-Line Emission in AGNs: The Effect of Prescription of the Gas Density" (2018). Physics and Astronomy Faculty Publications. 589.

https://uknowledge.uky.edu/physastron_facpub/589

This Article is brought to you for free and open access by the Physics and Astronomy at UKnowledge. It has been accepted for inclusion in Physics and Astronomy Faculty Publications by an authorized administrator of UKnowledge. For more information, please contact UKnowledge@lsv.uky.edu. 


\title{
Intermediate-Line Emission in AGNs: The Effect of Prescription of the Gas
}

Density

\author{
Digital Object Identifier (DOI) \\ https://doi.org/10.3847/1538-4357/aab350 \\ Notes/Citation Information \\ Published in The Astrophysical Journal, v. 856, no. 1, 78, p. 1-13. \\ (c) 2018. The American Astronomical Society. All rights reserved.
}

The copyright holder has granted the permission for posting the article here. 


\title{
Intermediate-line Emission in AGNs: The Effect of Prescription of the Gas Density
}

\author{
T. P. Adhikari ${ }^{1}\left(\mathbb{1}\right.$, K. Hryniewicz $^{1}$, A. Różańska ${ }^{1}$, B. Czerny ${ }^{2}$ (1) , and G. J. Ferland $^{1,3}{ }^{(1)}$ \\ ${ }^{1}$ Nicolaus Copernicus Astronomical Center, Polish Academy of Sciences, Bartycka 18, 00-716, Warsaw, Poland; tek@camk.edu.pl \\ ${ }_{3}^{2}$ Center for Theoretical Physics, Polish Academy of Sciences, Aleja Lotnikow 32/46, Warsaw, Poland \\ ${ }^{3}$ Department of Physics and Astronomy, The University of Kentucky, Lexington, KY 40506, USA \\ Received 2017 December 1; revised 2018 February 27; accepted 2018 February 27; published 2018 March 27
}

\begin{abstract}
The requirement of an intermediate-line component in the recently observed spectra of several active galactic nuclei (AGNs) points to the possible existence of a physically separate region between the broad-line region (BLR) and narrow-line region (NLR). In this paper we explore the emission from the intermediate-line region (ILR) by using photoionization simulations of the gas clouds distributed radially from the center of the AGN. The gas clouds span distances typical for the BLR, ILR, and NLR, and the appearance of dust at the sublimation radius is fully taken into account in our model. The structure of a single cloud is calculated under the assumption of constant pressure. We show that the slope of the power-law radial profile of the cloud density does not affect the existence of the ILR in major types of AGNs. We found that the low-ionization iron line, Fe II, appears to be highly sensitive to the presence of dust and therefore becomes a potential tracer of dust content in line-emitting regions. We show that the use of a disk-like cloud density profile computed for the upper part of the atmosphere of the accretion disk reproduces the observed properties of the line emissivities. In particular, the distance of the $\mathrm{H} \beta$ line inferred from our model agrees with that obtained from reverberation mapping studies in the Sy1 galaxy NGC 5548.
\end{abstract}

Key words: galaxies: active - methods: numerical - quasars: emission lines - radiative transfer

\section{Introduction}

Over the past several decades, the properties and origin of the broad-line region (BLR) as well as the narrow-line region (NLR) in active galactic nuclei (AGNs) have been extensively discussed in the literature (Davidson 1972; Krolik et al. 1981; Netzer 1990; Dopita et al. 2002; Czerny \& Hryniewicz 2011; Baskin et al. 2014; Czerny et al. 2015, 2017, and references therein). There is a general consensus that the two regions are physically separate and are spatially located at different distances from the central supermassive black hole (SMBH) of the AGN. The above conclusion arose naturally from the lack of any significant emission from lines with FWHM between $\sim 2000 \mathrm{~km} \mathrm{~s}^{-1}$, typical for a BLR, and $\sim 500 \mathrm{~km} \mathrm{~s}^{-1}$, typical for an NLR (Boroson \& Green 1992).

Theoretically, the lack of such emission from gas between the BLR and NLR was successfully explained by Netzer \& Laor (1993, hereafter NL93). The authors calculated the line emission from radially distributed clouds above an accretion disk, using photoionization computations. Each cloud was a slab of constant density illuminated by a quasar continuum of the same mean shape. The lack of line emission was successfully achieved by the introduction of dust. Practically, this means that the dust was taken into account in photoionization calculations for clouds located further away from the SMBH at a certain radius named the sublimation radius. Closer to the nucleus the radiation field is so strong that the dust grains cannot survive. The presence of dust for given gas conditions successfully suppresses line emission, and the gap between the BLR and NLR forms naturally. However, new observations with the largest instruments give us a new look at those objects.

There is a growing number of AGNs that exhibit emission lines with intermediate FWHM of $\sim 700-1200 \mathrm{~km} \mathrm{~s}^{-1}$ in their spectra, suggesting the existence of an intermediate-line region (ILR) in those sources. Brotherton et al. (1994) have defined an
ILR in 15 QSOs with broad UV lines as the second component of the BLR, located in the innermost part of the NLR.

Mason et al. (1996) found evidence for an ILR with velocity FWHM of $\sim 1000 \mathrm{~km} \mathrm{~s}^{-1}$ that produces a significant amount of flux in both permitted and forbidden lines in the ultrasoft X-ray source NLSy1 RE J1034+396. $\mathrm{H} \alpha, \mathrm{H} \beta$, and $\mathrm{O}[\mathrm{III}]$ were observed by the ISIS spectrograph on La Palma. Detailed spectral analysis of a large number of sources in the Sloan Digital Sky Survey (SDSS) has revealed the presence of an intermediate component of line emission with velocity width in between those of the broad and narrow components $(\mathrm{Hu}$ et al. 2008a, 2008b). SDSS sources show an ILR in $\mathrm{H} \alpha$ and $\mathrm{H} \beta$ lines (Zhu et al. 2009), mostly for NLSy1 galaxies.

For the Sy1 galaxy NGC 4151, Crenshaw \& Kraemer (2007) identified an intermediate-line emission component with width $\mathrm{FWHM}=1170 \mathrm{~km} \mathrm{~s}^{-1}$, most probably originating between the BLR and NLR. For the Sy1 galaxy NGC 5548, ILR was found to be located at $\sim 1 \mathrm{pc}$, with smaller velocity $\mathrm{FWHM}=$ $680 \mathrm{~km} \mathrm{~s}^{-1}$ (Crenshaw et al. 2009).

Moreover, the presence of an ILR in 33 galaxies with lowionization nuclear emission line regions (so called LINERs) was reported by Balmaverde et al. (2016) using HST/STIS (Hubble Space Telescope/Space Telescope Imaging Spectrograph). Since the typical obscuration torus is not present in LINERs, the authors suggest that the ILR takes the form of an ionized, optically thin torus. They also suggest that this tenuous structure is present only in LINERs because of the general paucity of gas and dust in their nuclear regions. This also causes their low rate of accretion and low bolometric luminosity.

Recently, Adhikari et al. (2016, hereafter AD16) studied the ILR by using photoionization simulations of ionized gas with dust in AGNs. Within the framework of the NL93 formalism, the authors found that in order to expect ILR emission the density of gas should be high, of the order of $\sim 10^{11.5} \mathrm{~cm}^{-3}$ at the sublimation radius, which means that for each cloud located 
at a certain distance the density in the AD16 paper was two orders of magnitude higher than in NL93. For such dense matter the gas opacities always dominate over the dust opacities in a region of line formation, and the dust cannot suppress the line emission as it did in the NL93 paper. Therefore, the usual gap between the BLR and NLR cannot be created, and an ILR can be present. The above result was achieved for three different spectral shapes of the illuminated continuum typical for Sy1.5, Sy1, and NLSy1 AGNs. In addition, AD16 argued that LINERs should also exhibit ILR emission, due to the low value of luminosity and therefore the ionization parameter in those sources.

The aim of this paper is to investigate in detail the physics of an ILR. We extend our previous studies (AD16) by (i) performing computations for a cloud model with constant pressure (CP) instead of constant density (CD), (ii) searching for the influence of a slope of the power-law density profile on the total line emission, (iii) including additional emission lines such as Fe II and C IV, (iv) considering the disk-like cloud density profile from the atmosphere of the accretion disk, (v) using self-consistent source luminosities and therefore the position of the sublimation radius.

We perform the photoionization simulations with the publicly available numerical code CLOUDY version C17.00 (Ferland et al. 2017), assuming that each cloud is under CP (Baskin et al. 2014). In the first step, we consider the model of a continuous cloud distribution above an accretion disk, following the approach of AD16. Nevertheless, we vary the power-law index that relates to the distribution of surface cloud density over the distance to the SMBH. We show that the existence of the ILR is not sensitive to the slope of the powerlaw density profile considered by us with the assumption of a $\mathrm{CP}$ cloud. The same result was obtained by Adhikari et al. (2017) in the case of CD clouds.

In the second step, we compute source luminosities for each of four sources, and we use those values to derive the position of the sublimation radius according to a formula given by Nenkova et al. (2008). This allows us implement dust correctly in our photoionization calculations. To achieve the physically consistent density profile, we assume that clouds are created from the upper parts of an accretion disk. By adopting black hole masses and accretion rates of the four considered sources, given in the literature, we simulate the vertical accretion disk structure assuming a standard disk (Shakura \& Sunyaev 1973) and the approximation of transfer of radiation by diffusion (Różańska et al. 1999). Furthermore, we employ the radial density profile obtained by solving the vertical accretion disk structure of a geometrically thin disk at optical thickness $\tau=2 / 3$ as described in Section 4. This can only be done by straight comparison of cloud density with the density of an upper disk atmosphere at a given radius. This idea is very close to the development made by Baskin \& Laor (2018), who associate the BLR directly with the accretion disk atmosphere.

For a disk-like cloud density profile, we obtained the prominent ILR in all four sources considered. However, the sublimation radius is $\sim 2$ orders of magnitude smaller for LINER NGC 1097 than the rest of the sources because of its low luminosity. The drop in the disk-like density profile causes mild enhancement of low-ionization lines (LILs), while highionization lines (HILs) are suppressed where the density drops. This result is in agreement with the two-component BLR model presented by Collin-Souffrin et al. (1988). The distance inferred from the time delay of $\mathrm{H} \beta$ in NGC 5548 taken from observations agrees with the distance at which the $\mathrm{H} \beta$ line peaks in our simulated line emissivity profile.

The structure of the paper is organized as follows. In Section 2, we describe the numerical model parameters taken for the photoionization computations. The whole of Section 3 is devoted to the effect of the slope of the power-law density profile on the computed line emission. In addition, the CP model is compared with the CD model explicitly in Section 3.1, while the dust-sensitive line Fe II is discussed in Section 3.4. Section 4 contains the results we obtained for the disk-like density prescription we adopted. Finally the discussion of line emission and conclusions are presented in Sections 5 and 6 respectively.

\section{Photoionization Simulations of the ILR}

In order to achieve the established properties of ionized gas located at different distances from the SMBH, which can be responsible for the observed broad- to narrow-line emission, we consider a distribution of clouds above an accretion disk, defined at each radial distance, $r$, by the gas density $n_{\mathrm{H}}$ at a cloud surface, total column density $N_{\mathrm{H}}$, and the chemical abundances. Photoionization processes are simulated with the numerical code CLOUDY version C17 (Ferland et al. 2017).

We used the CLOUDY default solar abundances derived by Grevesse \& Sauval (1998), for the gas clouds at distance $r \leqslant R_{\mathrm{d}}$, where we expect the BLR to be located. For the clouds at $r>R_{\mathrm{d}}$, where the NLR is supposed to occur, the composition of the interstellar medium (ISM) with dust grains is used. ${ }^{4}$ Depending on the radial density profile, we consider various values of sublimation radius $R_{\mathrm{d}}$, expressed below in Sections 3 and 4.

In all radially distributed cloud models, we adopt the profile for the column density after NL93 and AD16:

$$
N_{\mathrm{H}}(r)=10^{23.4}\left(r / R_{\mathrm{d}}\right)^{-1}\left[\mathrm{~cm}^{-2}\right] .
$$

The normalization value of column density, $N_{\mathrm{H}}=10^{23.4} \mathrm{~cm}^{-2}$, again is taken from NL93 and AD16 due to general agreement with the observed BLR column density.

For a given radial distance, the cloud pressure is kept constant (Baskin et al. 2014). In practice this means that the gas pressure increases with cloud optical depth as radiation pressure decreases exponentially with gas optical depth. This option of photoionization computations was incorporated in CLOUDY as radiation pressure confinement (RPC), and used by Baskin et al. (2014) for the purpose of studying the BLR. The concept of RPC does not differ from the total CP models used by Różańska et al. (2006, 2008) and Adhikari et al. (2015) in the case of the warm absorbers in AGNs. The only difference is in the numerical treatment of radiation pressure, which in the case of the latter authors is self-consistently computed from the true intensity of the radiation field (see Dumont et al. 2000 for description of the TITAN code). Nevertheless, for both approaches, the input parameter that is the hydrogen number density of an individual cloud, $n_{\mathrm{H}}$, is given only at the cloud surface. This is because the radiation pressure compresses the cloud, and the density gradient across the photoionized gas occurs naturally, which is self-consistently computed by the CLOUDY code. The exact comparison of CP and CD models regarding the ILR is given in Section 3.1.

\footnotetext{
See Hazy1 CLOUDY documentation for the details.
} 


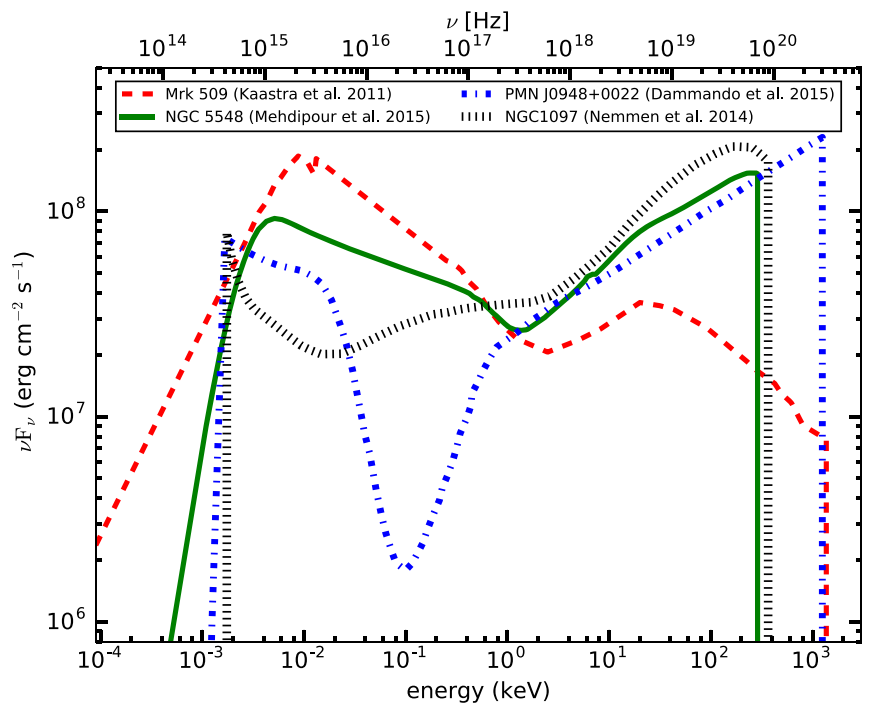

Figure 1. Shapes of the broadband spectra used in our photoionization calculations. In order to see the dependence on spectral shape all SEDs are normalized to $L_{\mathrm{bol}}=10^{45} \mathrm{erg} \mathrm{s}^{-1}$. See Table 1 for the exact values of luminosities.

For the radial distribution of clouds, the number density assumed at each cloud surface changes with distance from the SMBH according to a given radial profile of the density at the cloud surface $n_{\mathrm{H}}(r)$. The exact radial density profiles used in our computations are given in Sections 3 and 4 below.

The ionized clouds are distributed from $r=10^{-2}$ pc up to $r=10^{3} \mathrm{pc}$. For the given cloud location and for the given density at the cloud surface, the ionization parameter $U$ is computed by the CLOUDY code using the well known expression (Osterbrock \& Ferland 2006)

$$
U=\frac{Q_{\mathrm{H}}}{4 \pi r^{2} n_{\mathrm{H}} c},
$$

where $Q_{\mathrm{H}}$ is the number of hydrogen-ionizing photons in the incident radiation field and $c$ is the velocity of light.

In all our models of radially distributed clouds, we assume that each cloud is illuminated by a spectral energy distribution (SED) of the same shape. We consider four shapes of SED adopted from recent multi-wavelength observations of Sy1.5-Mrk 509 (Kaastra et al. 2011), Sy1-NGC 5548 (Mehdipour et al. 2015), NLSy1 galaxy PMN J0948+0022 (D'Ammando et al. 2015), and LINER-NGC 1097 (Nemmen et al. 2014), as displayed in Figure 1. In order to see the spectral shape of different types of AGN, we normalized all SEDs to $L_{\mathrm{bol}}=10^{45} \mathrm{erg} \mathrm{s}^{-1}$ in this figure. This value is used in all calculations presented in Section 3, while in Section 4 luminosities are taken directly from integrations of observations.

All final conclusions of our paper are based on analysis of the modeled line emissivity profiles given as an output of photoionization calculations. In the case of each source we present radial emissivity profiles for the most observed line transitions in the AGN spectra: $\mathrm{H} \beta \lambda 4861.36$, He II $\lambda 1640.00$, Mg II $\lambda 2798.0, \quad \mathrm{C}$ III] $\lambda 1909.00, \quad[\mathrm{O}$ III] $\lambda 5006.84, \quad$ Fe II $\lambda$ (4434-4684), and C IV $\lambda 1549.00$.

\section{Power-law Density Profile}

In order to investigate the influence of the density profile on the variation of emission line luminosity with radius, we assume in this section that the density at the cloud surface decreases with distance from the $\mathrm{SMBH}$ as

$$
n_{\mathrm{H}}=10^{11.5}\left(r / R_{\mathrm{d}}\right)^{-\beta} \quad\left[\mathrm{cm}^{-2}\right],
$$

where $\beta$ is the slope of the power-law density. The value of the density at the cloud surface at the sublimation radius, $R_{\mathrm{d}}$, (i.e., density normalization) is adopted after AD16. This is because AD16 have shown that it is only for such a high density value that an ILR can exist in the framework of this model. For all models computed in this section we adopt the same sublimation radius: $R_{\mathrm{d}}=0.1 \mathrm{pc}$, following NL93 and AD16.

For the density profile given by Equation (3), the resulting ionization parameter $U$ depends on the cloud location and on the number of ionizing photons (Equation (2)). Assuming the same bolometric luminosity, $L_{\mathrm{bol}}=10^{45} \mathrm{erg} \mathrm{s}^{-1}$, for each of the four sources, we obtain the following scaling laws of the ionization parameter with distance in pc:

$$
\begin{gathered}
U_{\text {Mrk } 509}=6.72 \times 10^{-6}\left(r / R_{\mathrm{d}}\right)^{\beta} r^{-2}, \\
U_{\text {NGC } 5548}=3.11 \times 10^{-6}\left(r / R_{\mathrm{d}}\right)^{\beta} r^{-2}, \\
U_{\text {NGC } 1097}=1.13 \times 10^{-6}\left(r / R_{\mathrm{d}}\right)^{\beta} r^{-2}, \\
U_{\text {PMNJ0948 }}=1.08 \times 10^{-6}\left(r / R_{\mathrm{d}}\right)^{\beta} r^{-2} .
\end{gathered}
$$

For the purpose of this paper, we consider three values of $\beta$, i.e., $0.5,1.5$, and 2.5 , which are chosen arbitrarily to relate density to different powers of distance. We note that $\beta=1.5$ converges to the profile used by NL93 and AD16.

\subsection{CP versus $C D$ for a Single Cloud}

We consider a single cloud in a spherically symmetric gravitational field, located at radial distance $r$ from the SMBH. We assume that locally the cloud thickness is negligible in comparison to the distance, which is equivalent to a locally plane-parallel approximation of the cloud geometry. The cloud is illuminated by radiative flux $F_{0}$ at the illuminated face $z_{0}$. The condition of hydrostatic equilibrium, for black hole mass $M_{\mathrm{BH}}$, with optical depth as a variable $d \tau=\kappa \rho d z$, is

$$
\frac{d P_{\mathrm{gas}}}{d \tau}=-\frac{1}{\kappa}\left(\frac{G M_{\mathrm{BH}}}{r^{2}}-\Omega^{2} r\right)-\frac{d P_{\mathrm{rad}}}{d \tau},
$$

where as usual $\rho$ is the volume density, $\kappa$ the mean opacity coefficient, $G$ the gravitational constant, $P_{\text {gas }}$ and $P_{\text {rad }}$ the gas and radiation pressures, and $\Omega$ the angular velocity of the gas. Expressing the radiation pressure gradient as a first-order solution of radiative transfer equtation, $d P_{\mathrm{rad}} / d \tau=-\left(F_{0} / c\right) e^{-\tau}$, and integrating over hydrostatic balance from 0 to $\tau$, we obtain

$$
\begin{aligned}
P_{\text {gas }}(\tau)= & P_{\text {gas }}(0)-\frac{1}{\kappa}\left(\frac{G M_{\mathrm{BH}}}{r^{2}}-\Omega^{2} r\right) \tau \\
& -\frac{F_{0}}{c} e^{-\tau}+\frac{F_{0}}{c},
\end{aligned}
$$

where $c$ is the velocity of light.

At the illuminated face of the cloud, the total pressure has some constant initial value: $C_{0}=P_{\text {gas }}(0)+F_{0} / c$. In addition the gas pressure gradient at the outer cloud surface $\left(\tau=\tau_{\max }\right)$ should be zero, since the cloud is finite and the radiation 
pressure and centrifugal force should balance the gravitational force there, i.e., $\left(G M_{\mathrm{BH}} / r^{2}-\Omega^{2} r\right) / \kappa=\left(F_{0} / c\right) e^{-\tau_{\max }}$. Adopting those conditions, the hydrostatic balance is

$$
P_{\text {gas }}(\tau)=C_{0}-\frac{F_{0}}{c}\left(e^{-\tau}+\tau e^{-\tau_{\max }}\right) .
$$

To put limits on the initial conditions of cloud pressure that satisfy hydrostatic balance we express the ratio of gas pressures in the two extreme cases, i.e., for $\tau=0$ and $\tau=\tau_{\max }$ :

$$
\frac{P_{\mathrm{gas}}\left(\tau_{\max }\right)}{P_{\mathrm{gas}}(0)}=\frac{C_{0}-\frac{F_{0}}{c} e^{-\tau_{\max }}\left(1+\tau_{\max }\right)}{C_{0}-\frac{F_{0}}{c}} .
$$

The above equation does not set any requirement for the initial values of cloud pressure to be in pressure equilibrium. We can consider special limits. Assuming $\tau_{\max } \ll 1$, we get

$$
\frac{P_{\text {gas }}\left(\tau_{\max }\right)}{P_{\text {gas }}(0)}=1 ; \Rightarrow P_{\text {gas }}=\text { const },
$$

which is the well known case of a cloud with constant gas pressure. This means that for optically thin clouds we do not have strong modification of the gas pressure by the radiation pressure. The second special case occurs for $\tau_{\max } \gg 1$, and we get

$$
\frac{P_{\mathrm{gas}}\left(\tau_{\max }\right)}{P_{\mathrm{gas}}(0)}=\frac{C_{0}}{C_{0}-\frac{F_{0}}{c}}=1+\frac{P_{\mathrm{rad}}(0)}{P_{\mathrm{gas}}(0)} .
$$

In this case the density gradient inside the cloud depends on the adopted value of the ratio of gas pressure to radiation pressure at the illuminated face of cloud. When $P_{\text {rad }}(0) \ll P_{\text {gas }}(0)$ we again get the condition of the cloud being under constant gas pressure. But when $P_{\text {rad }}(0) \geqslant P_{\text {gas }}(0)$, the compression of the cloud by radiation pressure is always present and increases with increasing value of this ratio. Thus the requirement that radiation pressure should be much larger than the gas pressure, made by Baskin et al. (2014) for the RPC model, is only a special case among solutions for the CP cloud, where compression is the strongest. Physically, this is always the case for warm absorbers in AGNs modeled by Różańska et al. (2008). Nevertheless, many other solutions of clouds being under constant total pressure are possible, from small compression equivalent to the model of constant gas pressure up to strong RPC as considered by Baskin et al. (2014) in the case of the BLR.

The physical conditions considered in this paper, needed to give strong emission at the ILR, put us into the limit of $P_{\text {rad }}(0)<P_{\text {gas }}(0)$ by roughly two orders of magnitude depending on the cloud location. However, constant gas pressure even in this case is not equivalent to the CD model, since even constant gas pressure can imply a large density and temperature gradient. To illustrate the difference between $\mathrm{CP}$ and $\mathrm{CD}$ clouds within the framework of our model we present in Figure 2 the structure of a single cloud as a result of photoionization calculations with the CLOUDY code. Our cloud is located at $1 \mathrm{pc}$ from the SMBH and illuminated by the SED of Mrk 509. The assumed density on the cloud surface is of the order of $n_{\mathrm{H}}=10^{10} \mathrm{~cm}^{-3}$. The difference between the assumptions of constant density (dashed blue line) and CP (solid red line) is noticeable. The density structure for the $\mathrm{CP}$ cloud is not
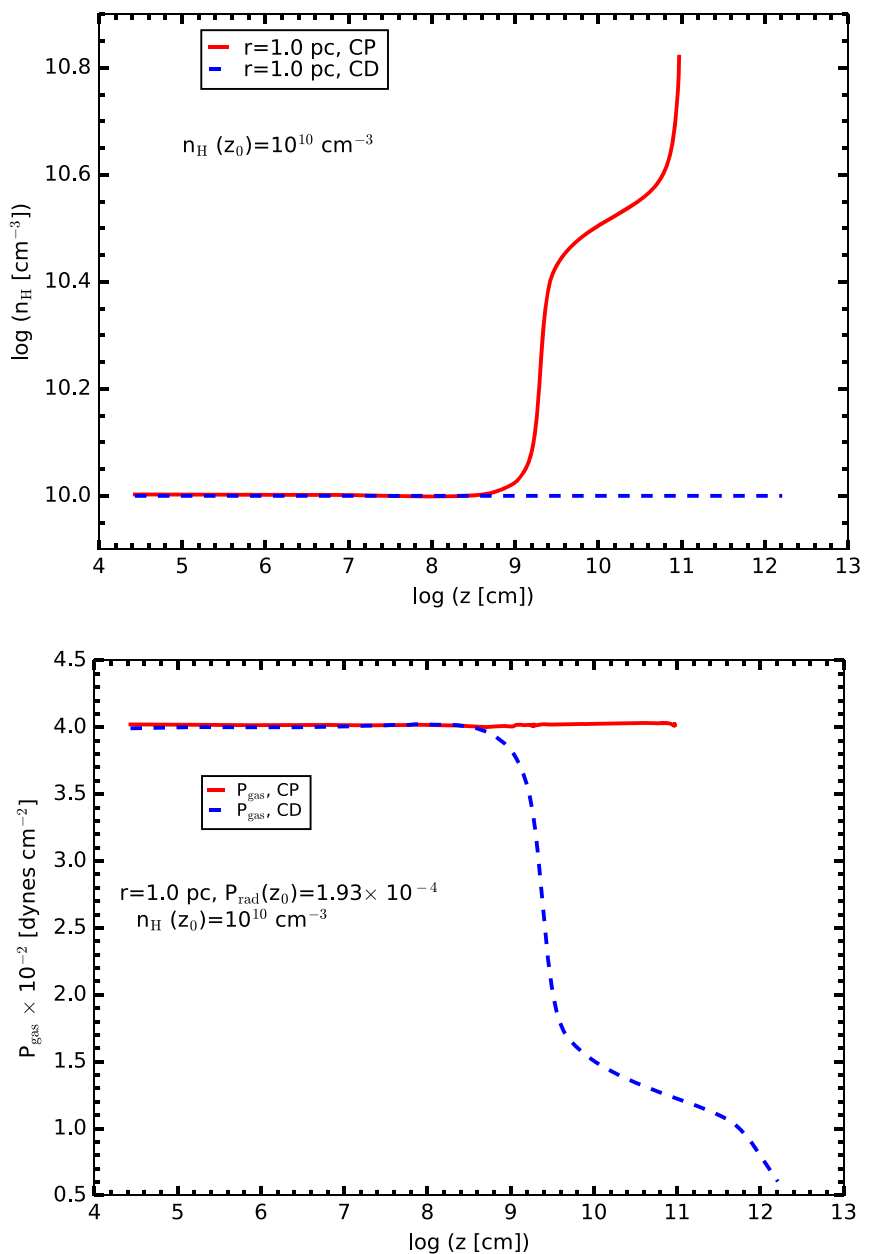

Figure 2. The comparison of models of constant density (CD) and constant pressure (CP) for a single cloud with a density of $n_{\mathrm{H}}=10^{10} \mathrm{~cm}^{-3}$ at the illuminated cloud surface. The SED of Mrk 509 is used in both simulations. The stratifications in density and gas pressure are shown in the upper and lower panels, respectively.

constant, even when compression is very weak, since radiation pressure is gradually absorbed with cloud optical depth.

Lower gas pressure at the illuminated cloud surface can relatively increase the compression by radiation pressure. Physically we can achieve this condition for lower density. In Figure 3 we present the same single-cloud comparison for density of the order of $10^{5} \mathrm{~cm}^{-3}$. For this case we are in the limit where $P_{\text {rad }}(0) \approx P_{\text {gas }}(0)$ and compression is clearly visible as a rise in density and gas pressure with cloud thickness. Nevertheless, recently we have shown that an ILR can exist only if density is high (Adhikari et al. 2016), which means that our CP clouds are not too strongly compressed, and therefore not so different from the CD model.

The requirement of a high-density cloud at the sublimation radius sets the sound-crossing timescale to be two orders of magnitude smaller than the dynamical timescale, which is expected to be a few years at $R_{\mathrm{d}}=0.1 \mathrm{pc}$. The cooling/heating timescale for such dense gas is even several orders of magnitude shorter than the sound-crossing timescale; therefore we assume that clouds are in both thermal and hydrostatic equilibrium. With such an assumption, even dense clouds can survive for at least a fraction of the local Keplerian period without being destroyed. 

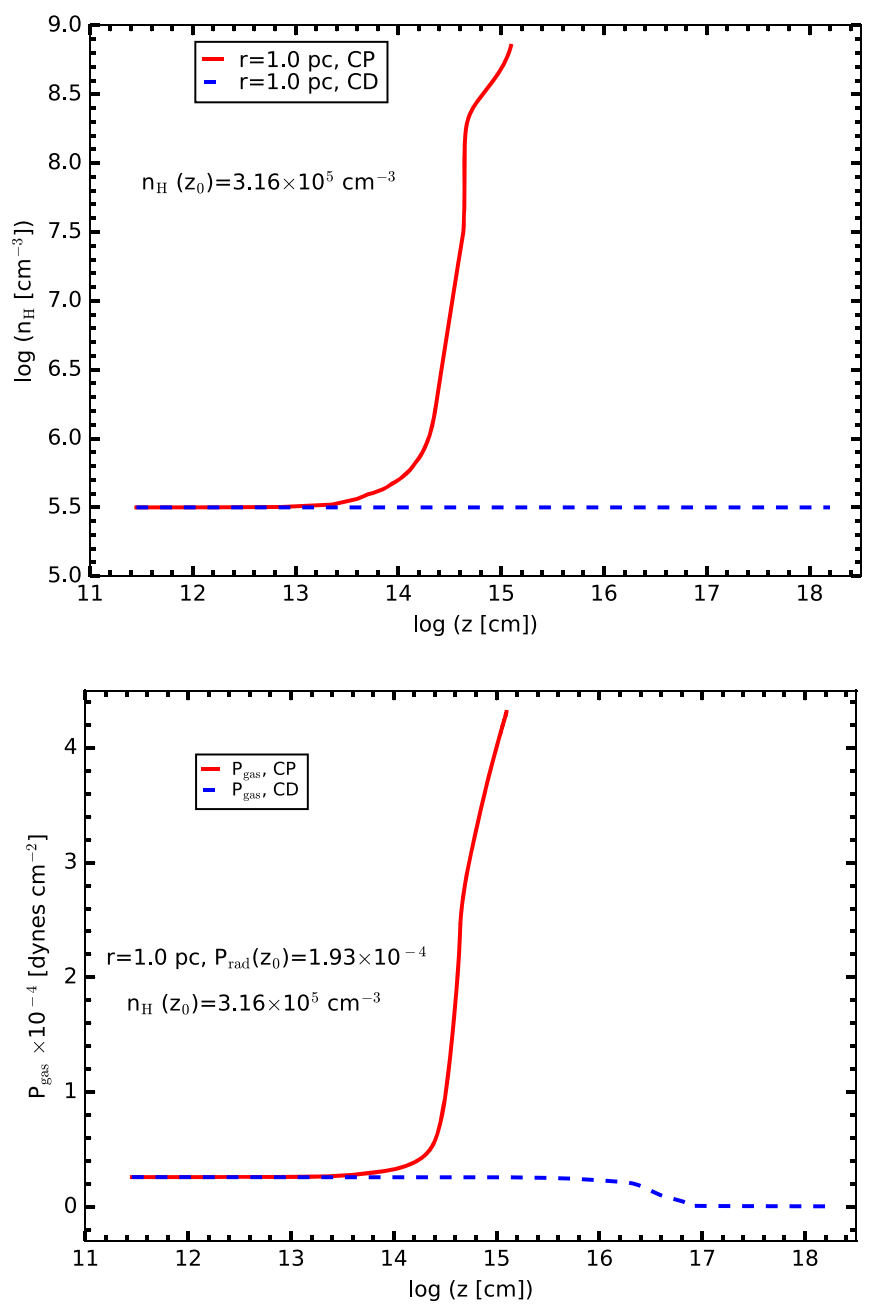

Figure 3. The same as in Figure 2, but for a low-density cloud with $n_{\mathrm{H}}=3.16 \times 10^{5} \mathrm{~cm}^{-3}$ at the illuminated cloud surface.

\section{2. $C P$ versus $C D$ for the Full Model}

With the above considerations, we derived the line luminosities for the major emission lines, which we present in Figure 4 for the case of the spectral shape of the Sy1.5 galaxy Mrk 509. For better visibility we draw emission from LILs$\mathrm{H} \beta, \mathrm{Mg}$ II, and Fe II-in the two leftmost columns, while other HILs-He II, C III], [O III], and C IV-are shown in the two rightmost columns.

Each pair of columns in Figure 4 represents a comparison between the model that assumes that each cloud is computed under constant density and the model that assumes CP clouds. We can easily see that emission line luminosities do not differ when the more physical model of CP is used. The profiles of all emission lines are practically the same when we compare left and right in each pair of columns. We demonstrate here that for such colder clouds as in the BLR and NLR, the compression by radiation pressure is not as important as in warm absorbers studied by Różańska et al. (2006) and Adhikari et al. (2015). As shown in the previous subsection, we may expect some differences for lower density normalization at a sublimation radius. But for lower density we are not able to produce the visible ILR region, which is one purpose of this paper. The above conclusion is valid for all four spectral shapes used in this paper; therefore for other AGN types we present only $\mathrm{CP}$ models of line emissivity in Figures 5-7.

\subsection{The Slope of the Power-law Density}

Only for $\beta=1.5$ do the overall line emissivity profiles stay flat along the radius in the case of the four AGN types, with the exception of Fe II and C IV lines. The density profile with such a power-law slope changes from $\sim 10^{12} \mathrm{~cm}^{-3}$ in the BLR to $\sim 10^{6} \mathrm{~cm}^{-3}$ in the NLR, with the latter value favored by narrow lines.

The situation changes when $\beta=0.5$, and the density is still high at $\sim 10^{9.5} \mathrm{~cm}^{-3}$ in the NLR. All LILs such as $\mathrm{H}_{\beta}, \mathrm{Mg}$ II, and Fe II (always presented in panels in the left column) display a sudden drop in emissivity in the NLR range $r \gtrsim 100 \mathrm{pc}$, caused by the low value of the ionization parameter (due to high density). The exception is Mrk 509 (Figure 4), because there are many UV photons in its spectral shape (Figure 1, dashed red line). These many photons still keep the ionization parameter high enough to produce strong LILs. On the other hand, HILs (always presented in panels in the right column) decrease monotonically with distance.

At the second extremum, when $\beta=2.5$, the density is very low, $\sim 10^{1.5} \mathrm{~cm}^{-3}$, in the NLR. This provides the visible drop in LILs in the NLR range $r \gtrsim 100 \mathrm{pc}$, caused by too low a density. It happens in all types of AGN. Nevertheless, the emission of HILs increases with distance from SMBH up to about $r \sim 10 \mathrm{pc}$ due to a relatively high ionization parameter. Further away from the center, such emission becomes flat or decreases, depending on the value of the ionization parameter.

In general the results do not depend much on SED shape, and in all cases the ILR is visible with the exception of Fe II permitted and [O III] forbidden lines. Forbidden lines are effectively produced in a low-density environment, and for many cases presented here their emissivity is too low.

\subsection{Dust-sensitive Fe II Line}

In all cases, the Fe II line is the only line that shows a strong drop in emissivity by several orders of magnitude at the sublimation radius. Such behavior predicts the lack of an intermediate component for this line. Therefore, based on the results of our simulations, the Fe II line is sensitive to the presence of dust, and it is not an ILR indicator.

In our model, the strong drop in Fe II emissivity may be caused by two effects. The first one is the presence of dust discussed above, but the second effect can be the change in abundances in the gas phase when passing the sublimation radius. The assumed solar composition for $r<R_{\mathrm{d}}$ has an iron abundance two orders of magnitude higher than the ISM composition for $r>R_{\mathrm{d}}$. All other elements display abundances of the same magnitude when changing from solar to ISM composition. The change in abundances mimics the depletion of metals due to dust sublimation, as was already assumed by NL93 and AD16. In order to check what really causes the strong drop in the Fe II emissivity profile at $R_{\mathrm{d}}$, we made a test with different abundances in dustless clouds located at the BLR. Results are presented in Figure 8, where we compare two models. In the first model, we assume the ISM chemical composition with no grains for BLR clouds, while in the second model, the same clouds have typical solar abundances. In both models the ISM composition with grains is used for dusty clouds located further out than $R_{\mathrm{d}}$. It is clearly seen that the dust present is responsible for the drop in Fe II emissivity by about two orders of magnitude, while the change in iron abundance enhances this effect by one order of magnitude. 


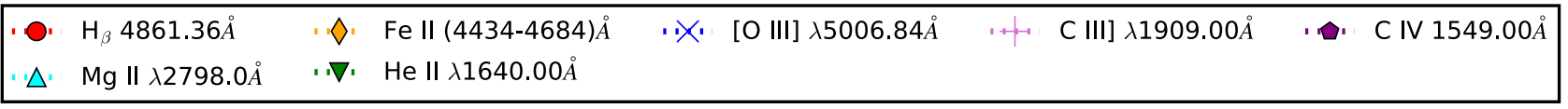

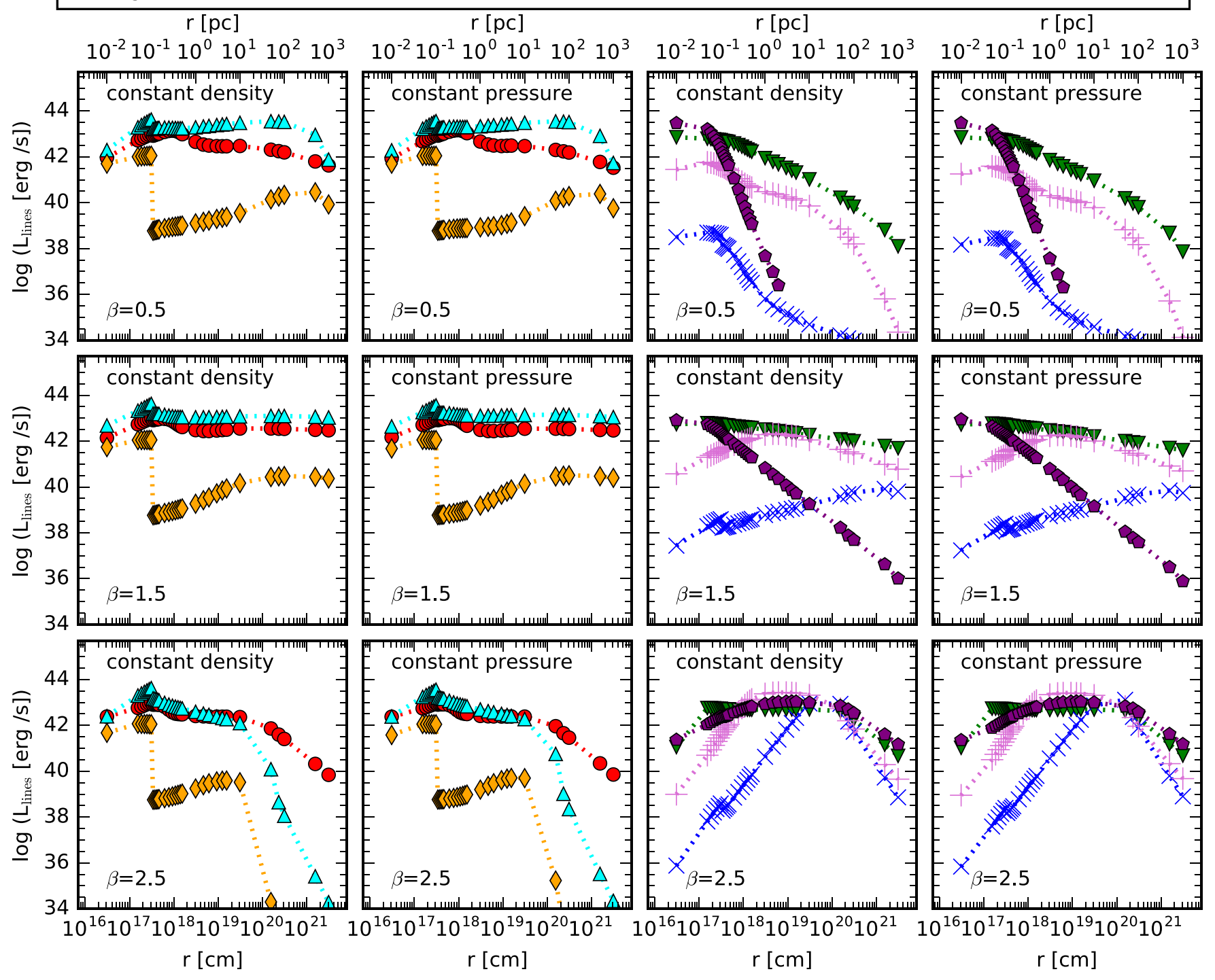

Figure 4. Line luminosity vs. radius for the Sy1.5 SED of Mrk 509. The two leftmost columns represent LILs: H $\beta$, Mg II, and Fe II lines for the cases of constant density and constant pressure respectively, while the two rightmost columns show HILs: He II, C III], [O III], and C IV lines again for constant density and constant pressure respectively. Panels in the three rows show the cases for $\beta=0.5,1.5$, and 2.5 from top to bottom.

\section{Disk-like Density Profiles}

Many years ago it was postulated that at different radii we should have outflows from a disk atmosphere in AGNs (i.e., Elvis 2004). In this section we consider a disk-like density profile, $n_{\mathrm{H}}(r)$, which is expected where clouds are formed from outflowing gas above the accretion disk atmosphere. We do not specify the mechanism that forms clouds, we just assume they do exist and that they should have the same density as the upper disk atmosphere. To determine the disk-like density profile of the cloud, we have to specify disk parameters, such as black hole mass and accretion rate, for each type of AGN. Table 1 displays all the important values used in our further computations.

By adopting black hole masses and accretion rates, we simulate the vertical structure of the accretion disk assuming a standard disk (Shakura \& Sunyaev 1973) and the approximation of transfer of radiation by diffusion, with gray gas opacities as described by Różańska et al. (1999). Furthermore, we employ the radial density profile of the cloud with the requirement that the adopted gas density at the illuminated surface of the cloud is equal to the density of the disk atmosphere where $\tau=2 / 3$, i.e., the atmosphere is still optically thick. When this comparison is made at each distance from the black hole, we obtain a disk-like cloud density profile.

The disk-like density profiles for all sources are presented in Figure 9. Such radial density profiles possess the characteristic feature of a strong rise density up to $10^{15} \mathrm{~cm}^{-3}$, located around the position of the BLR, $r \sim 10^{-2}$ pc. This is caused by a strong hump in opacity in an accretion disk atmosphere. Outside the hump the density declines from about $10^{13} \mathrm{~cm}^{-3}$ at the distance of $10^{15} \mathrm{~cm}$ from black hole, to about a few times $10^{9} \mathrm{~cm}^{-3}$ at greater distances of $10^{19} \mathrm{~cm}$. The corresponding ionization parameters for each disk-like density profile are presented in Figure 10. One can see a noticeable difference in the degree of ionization, which is four orders of magnitude lower for the LINER than for Sy1 and NLSy1. In addition, the 

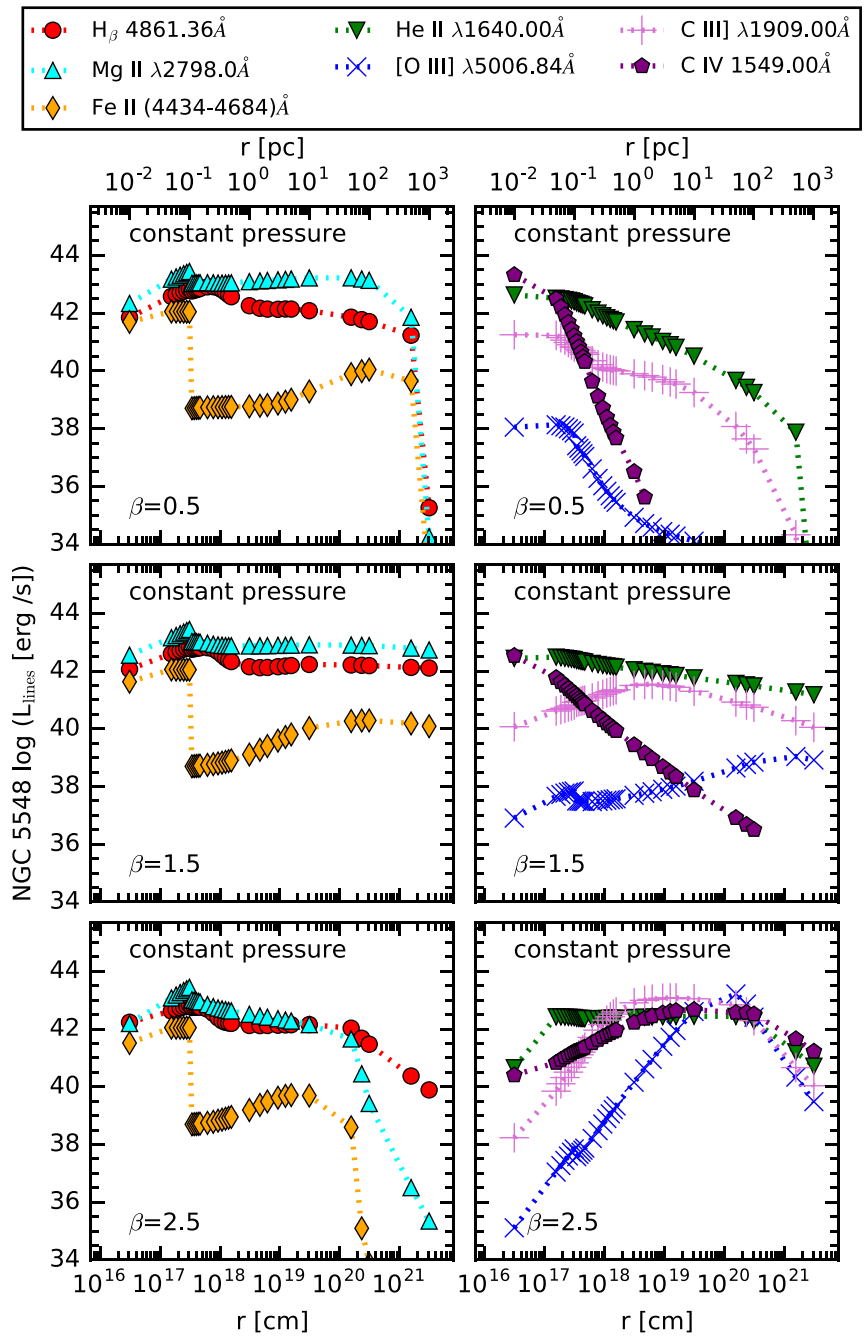

Figure 5. Line luminosity vs. radius for the Sy1 SED of NGC 5548. Panels in the left column represent LILs: $\mathrm{H} \beta, \mathrm{Mg}$ II, and Fe II, while the right column shows HILs: He II, C III], [O III], and C IV, for the model with constant pressure. The three rows show the cases for $\beta=0.5,1.5$, and 2.5 from top to bottom.

hump in density is directly reflected in the drop in ionization in all sources.

In this section, the source luminosity, $L$, given in the third column of Table 1 is obtained by integrating the flux over the energy range from 1 to $10^{5} \mathrm{eV}$. Furthermore, the dust sublimation radius, $R_{\mathrm{d}}$, for each source luminosity is computed using the following formula given by Nenkova et al. (2008):

$$
R_{\mathrm{d}}=0.4 \sqrt{L / 10^{45}} \quad[\mathrm{pc}] .
$$

This formula simply indicates the radius at which, for a given luminosity, the gas temperature reaches $1400 \mathrm{~K}$. Below this temperature dust can survive as a substantial gas component. The sublimation radius corresponding to each type of AGN is given in the sixth column of Table 1. It is clear that the luminosity influences the position of the sublimation radius, which we fully take into account in this section. In addition, the normalization of $N_{\mathrm{H}}$ to $10^{23.4} \mathrm{~cm}^{-3}$ differs for each source, since it is set at the position of the sublimation radius.

The position of the sublimation radius depends strongly on the detailed dust composition, which is still under discussion (Gaskell 2017; Xie et al. 2017). The dust is most likely a
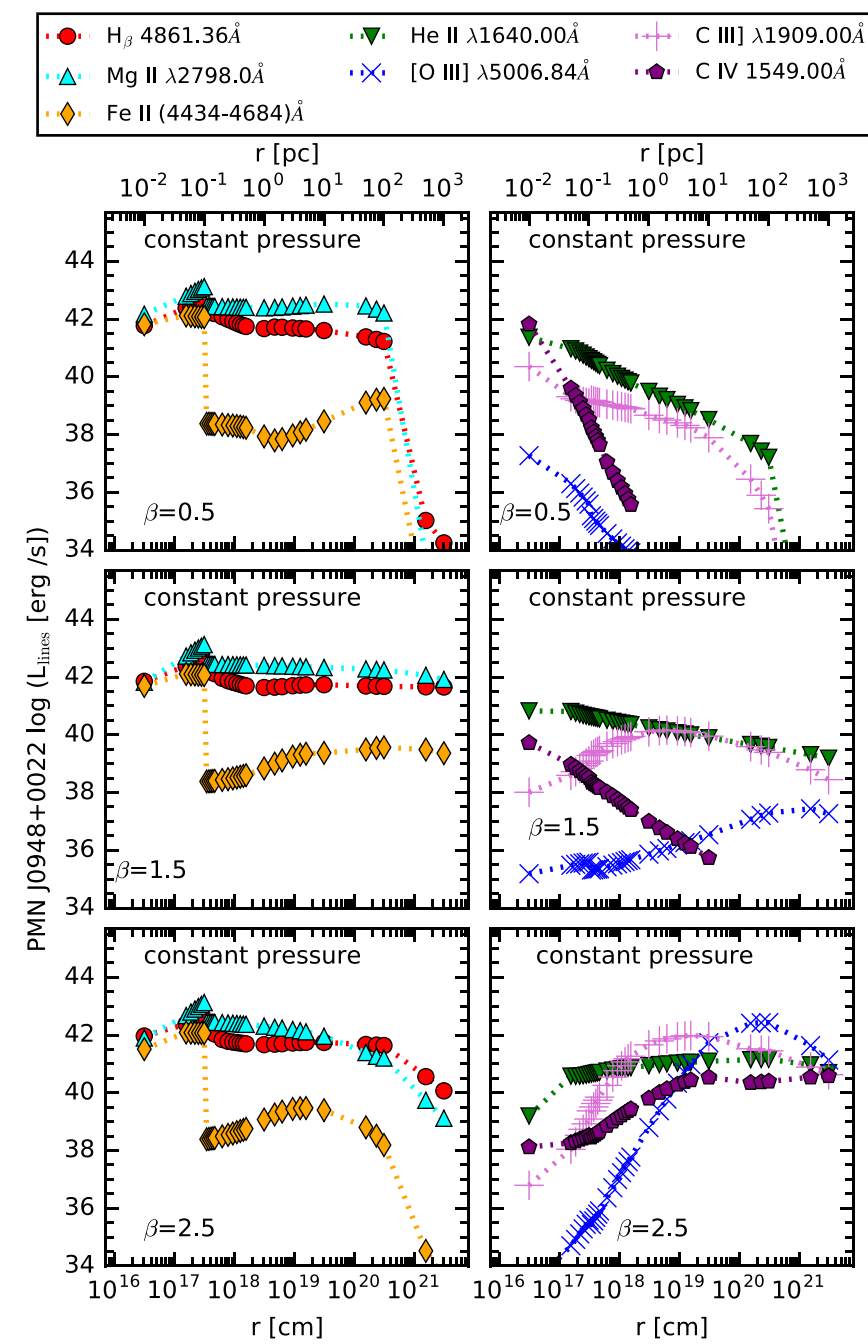

Figure 6. The same as in Figure 5, but for the NLSy1 SED of PMN J0948+0022.

mixture of amorphous carbon (Czerny et al. 2004), silicate (Lyu et al. 2014), and graphite grains (Baskin \& Laor 2018), while the sublimation radius derived by Nenkova et al. (2008) corresponds to the temperature of sublimation of silicate grains only (Laor \& Draine 1993). Graphite grains sublimate at a higher temperature of up to 2000 K (Laor \& Draine 1993; Baskin \& Laor 2018); nevertheless AGN extinction curves do not show the $2175 \mathrm{~A}$ carbon feature (Maiolino et al. 2001), which makes the dust in the circumnuclear region of AGNs different from the Galactic ISM. Nevertheless, to show how our results do depend on the dust sublimation radius, we present our model computed for two sources with a sublimation radius about 10 times smaller: $R_{\mathrm{d}}=0.06 \sqrt{L / 10^{45}} \mathrm{pc}$, which corresponds to the sublimation temperature of graphite (Laor \& Draine 1993).

We assume that emitting clouds emerge directly from the disk's atmosphere and preserve its density over the whole range of radii. This is a reasonable assumption at least for the low-ionization part of the BLR because it may develop as a failed wind embedded in the disk's atmosphere (Czerny \& Hryniewicz 2011). On the other hand, this assumption is the same as the model of the BLR being a part of the accretion disk atmosphere (Baskin \& Laor 2018). Our approach is not in contradiction with the geometry of a line-emitting medium 

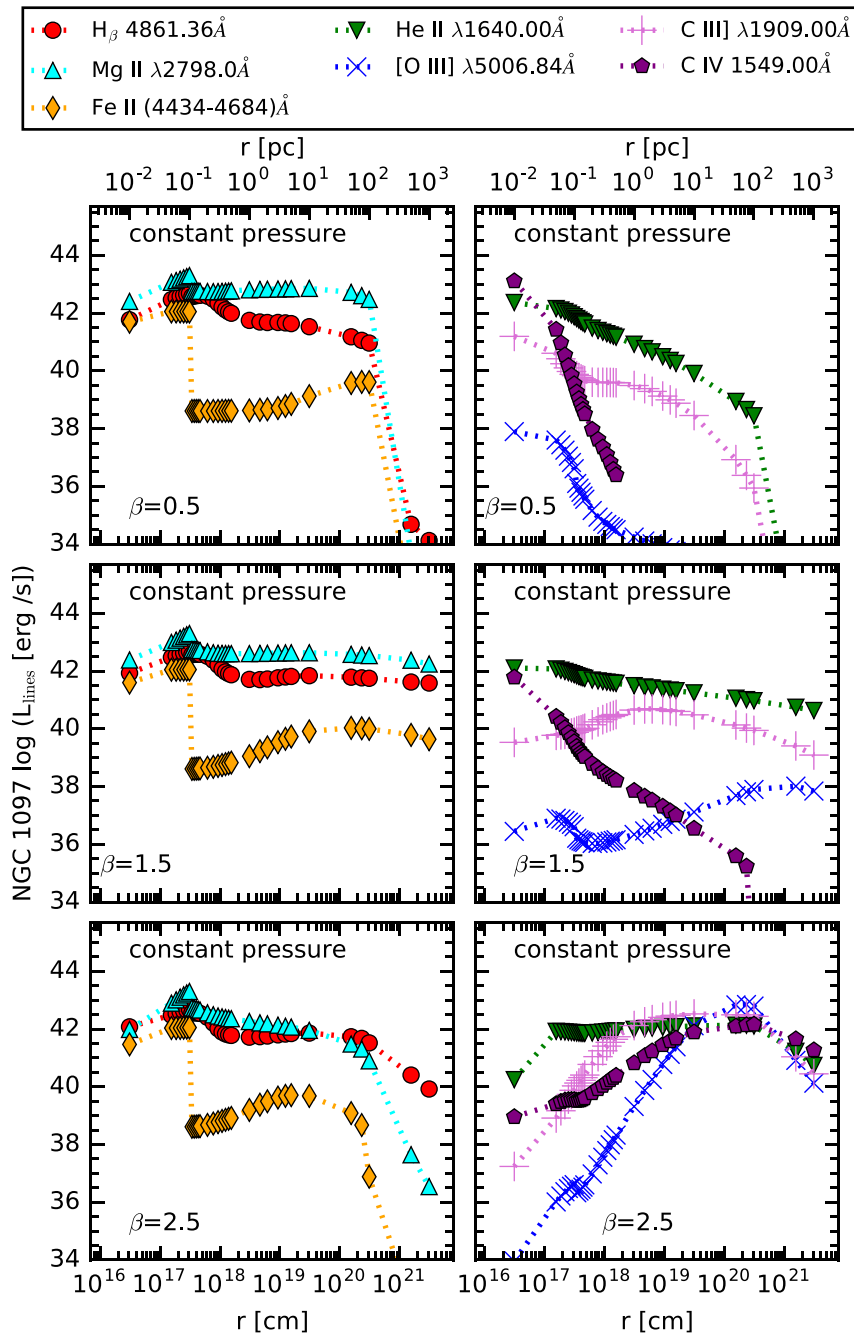

Figure 7. The same as in Figure 5, but for the LINER SED of NGC 1097.

similar to a bowl on top of the accretion disk (Gaskell 2009; Goad et al. 2012).

The resulting line emissivity profiles for four sources are presented in Figure 11 for HILs and LILs, and in Figure 12 for only LILs. In addition, for each source we show the total dust and total gas emission as a magenta solid line and black dashed line respectively. Shaded areas on the figures mark the positions of the BLR, ILR, and NLR, which depend on the AGN type. Assuming that all emitting gas is dominated by Keplerian motion, the BLR marked with a pink shade ranges from $15,000 \mathrm{~km} \mathrm{~s}^{-1}$ down to $3000 \mathrm{~km} \mathrm{~s}^{-1}$. The ILR marked with a green shade ranges between 3000 and $900 \mathrm{~km} \mathrm{~s}^{-1}$, while the NLR marked with a violet shade ranges from $900 \mathrm{~km} \mathrm{~s}^{-1}$ down to $300 \mathrm{~km} \mathrm{~s}^{-1}$.

In all AGN types, the emissivity profiles of LILs are insensitive to the hump in density in the disk-like cloud density profile. On the other hand, HILs display a strong drop in luminosity that reflects the density enhancement in the cloud radial profile. Such a drop in HIL luminosity is usually situated in the BLR, indicating the division of the BLR into the two types of low and high ionization as previously suggested by Collin-Souffrin et al. (1988), Collin et al. (2006), and Czerny \& Hryniewicz (2011).

In the case of PMN J0948 and Mrk 509, the luminosity is high enough to push the sublimation radius above $0.1 \mathrm{pc}$,

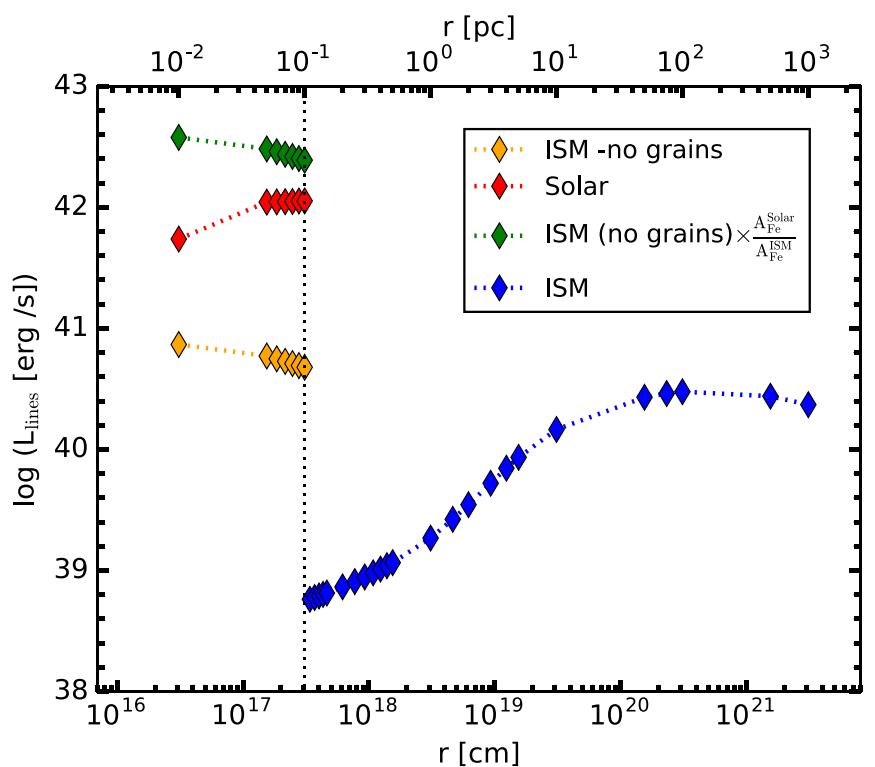

Figure 8. Comparison of Fe II line luminosity for two models of clouds illuminated by the Mrk 509 SED with different abundances of dustless clouds. The ISM composition with grains is used for dusty clouds located further out than $R_{\mathrm{d}}$, marked by the vertical dotted line. For clouds located closer to the SMBH than $R_{\mathrm{d}}$ we plotted Fe II line luminosity as red diamonds for solar iron abundance and as orange diamonds for the ISM abundance without grains. Green diamonds mark the ISM model without grains multiplied by the $\mathrm{Fe}$ abundance ratio of those two models.

Table 1

Parameters used in Computations of Disk-like Density Profile and the Position of $R_{\mathrm{d}}$

\begin{tabular}{llcccc}
\hline \hline Name & AGN & $\begin{array}{c}L_{\left(1-10^{5}\right) \mathrm{eV}} \\
\left(\mathrm{erg} \mathrm{s}^{-1}\right)\end{array}$ & $\begin{array}{c}M_{7}^{\mathrm{BH}} \\
\left(M_{\odot}\right)\end{array}$ & $\begin{array}{c}\dot{m} \\
\left(\dot{M}_{\text {Edd }}\right)\end{array}$ & $\begin{array}{c}R_{17}^{\mathrm{d}} \\
(\mathrm{cm})\end{array}$ \\
\hline Mrk 509 & Sy1.5 & $6.62 \times 10^{45}$ & $14^{\mathrm{a}}$ & $0.30^{\mathrm{b}}$ & 31.6 \\
NGC 5548 & Sy1 & $1.28 \times 10^{44}$ & $6.54^{\mathrm{c}}$ & $0.02^{\mathrm{d}}$ & 4.41 \\
PMN J0948 & NLSy1 & $2.28 \times 10^{46}$ & $15.4^{\mathrm{e}}$ & $0.40^{\mathrm{f}}$ & 58.9 \\
NGC 1097 & LINER & $9.62 \times 10^{40}$ & $14^{\mathrm{g}}$ & $0.0064^{\mathrm{h}}$ & 0.12 \\
\hline
\end{tabular}

Notes. The first and second columns list the name of the AGN and its type. The integrated luminosity is given in the third column. The black hole masses in units of $10^{7} M_{\odot}$, and accretion rates in units of Eddington accretion rate, follow in the fourth and fifth column respectively. Both values are taken from references listed below. The derived dust sublimation radius in units of $10^{17} \mathrm{~cm}$ is given in the last column.

${ }^{a}$ Mehdipour et al. (2011).

b Boissay et al. (2014).

c Bentz et al. (2007).

d Crenshaw et al. (2009), Ho \& Kim (2014).

e Foschini et al. (2011).

f Abdo et al. (2009).

g Onishi et al. (2015).

${ }^{\mathrm{h}}$ Nemmen et al. (2014).

making part of the ILR free from dust. This allows the maximum emissivity to appear within the ILR below the sublimation radius (three upper panels of Figure 11). Thus, in Seyferts, our model predicts a dominating intermediate component in LILs: $\mathrm{H} \beta, \quad \mathrm{Mg}$ II, and Fe II. Their line luminosities rise monotonically up to the sublimation radius. Nevertheless, the emission of HILs-He II, C III], [O III], and $\mathrm{C}$ IV-is maximal in the outer BLR or inner ILR, and decreases with distance. 


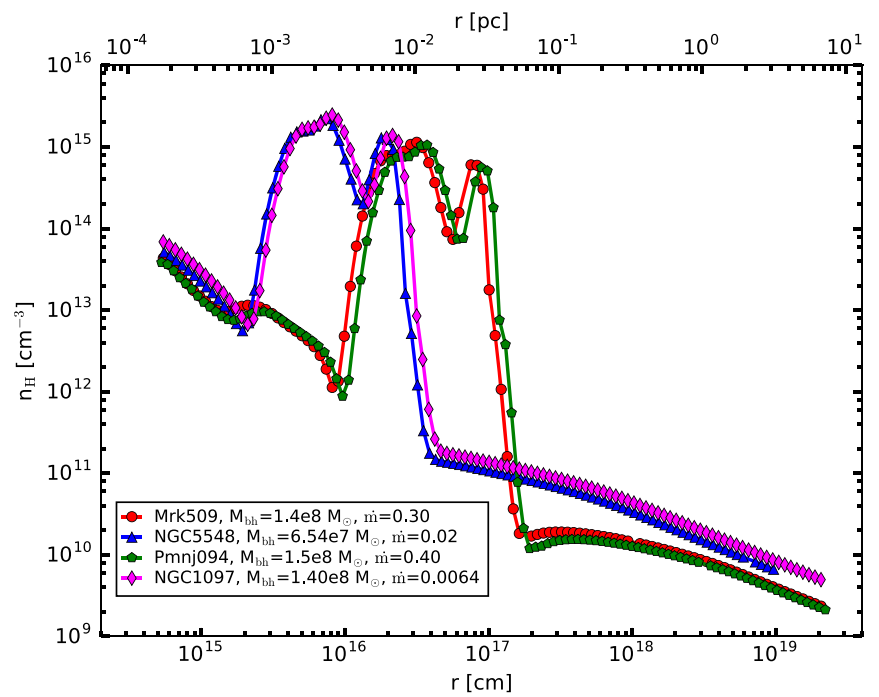

Figure 9. Radial density profiles as expected from the upper zones of the accretion disk atmospheres for all considered sources: parameters used in computations of the corresponding density profiles are also shown in the legend box.

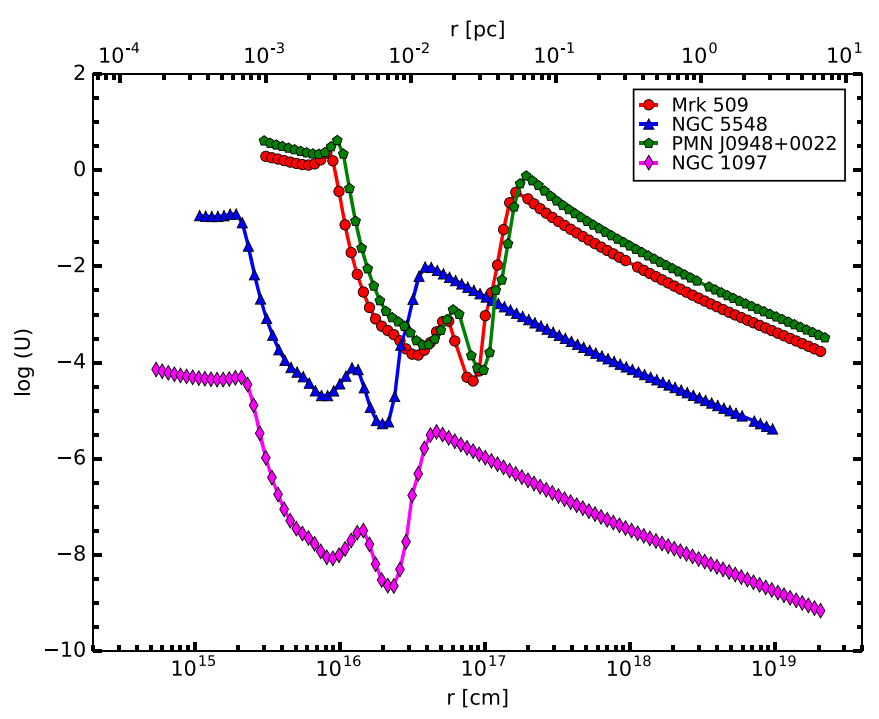

Figure 10. Profiles of radial ionization parameter $U$ at the cloud surface computed with Equation (2) for disk-like density profiles as shown in Figure 9.

In the case of a LINER, the dust sublimation radius appears at the position of the drop in HIL emissivity, caused by the hump in cloud density, because of its low luminosity. The presence of dust in the BLR provides flat line emissivities all the way to the NLR. Therefore, an ILR could be present in a LINER, but it is not as strong as in other types of AGN. Our result confirms the statement made by Balmaverde et al. (2016), where the authors concluded that a high-density component of the inner portion of the ILR is visible in Seyferts whereas the entirety of ILR emission is visible in a LINER. Nevertheless, the density of ILR clouds in a LINER inferred by those authors is $10^{4-6} \mathrm{~cm}^{-3}, \sim 5$ orders of magnitude less than the density of the ILR clouds in our model. We predict the location of the ILR in NGC 1097 to be in the range of radii $0.07-0.8 \mathrm{pc}$, whereas the distance of the ILR inferred by Balmaverde et al. (2016) is $1-10 \mathrm{pc}$.

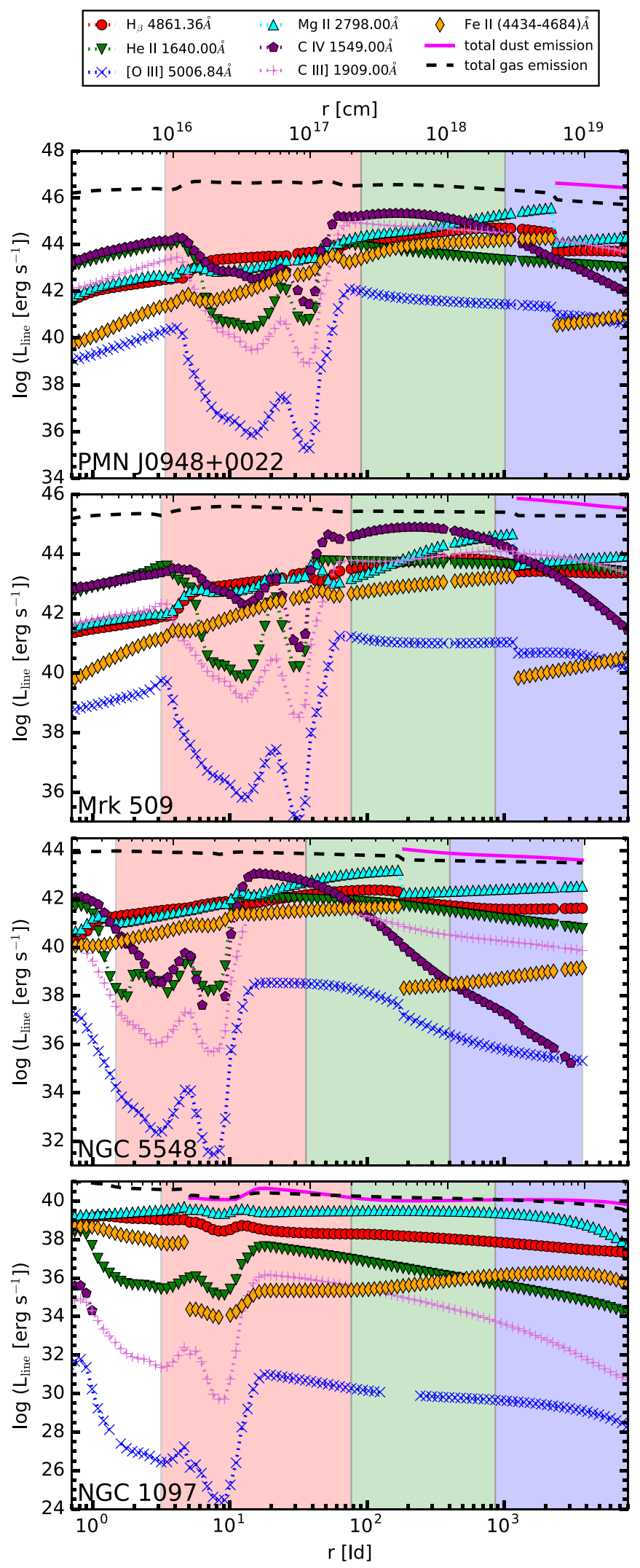

Figure 11. Luminosities of LILs and HILs vs. radius (in light-days) obtained for disk-like cloud density profiles. Each panel shows one type of AGN such that the source luminosity (given in Table 1) decreases from the top to the bottom panel. Shaded areas mark the positions of the BLR, ILR, and NLR from left to right, based on the adopted range of Keplerian velocities (see text for details). 
In general, for a disk-like density profile, additional lines such as $\mathrm{Mg}$ II and $\mathrm{H} \beta$ (slightly) appear to be dust-sensitive. Their luminosity profiles exhibit a rapid decrease when dust appears in clouds located relatively far from the center. This does not happen in the case of a LINER, since dusty clouds are still very dense (Figure 12, fourth panel).

The model of a disk-like cloud density distribution is less general than the model of an arbitrarily chosen density profile in locally optimal clouds (Goad \& Korista 2014), but it depends heavily on the conditions in the disk's upper atmosphere. The results are also different. While in our model the emissivity of the $\mathrm{H} \beta$ line increases with distance by two orders of magnitude for Seyfert AGNs, Goad \& Korista (2014) have shown a decline of this line between 1 and 100 light-days. The consequence would be that our model predicts a greater importance of the ILR component.

\section{Emission Line Regions}

The Seyfert 1 galaxy NGC 5548 is one of the best studied AGNs. It has been regularly monitored for almost four decades. Thus it is clear that a model of this source could be discussed most critically. We can compare approximate distances of emitting regions from our model with the results of reverberation mapping campaigns. We are aware that reverberation mapping depends on measurement of the continuum luminosity (Peterson et al. 2004; Bentz et al. 2006, 2007; Denney et al. 2009), and data show that measurements of delays in $\mathrm{H} \beta$ span over 6-30 days depending on the continuum luminosity. Therefore, in the case of the $\mathrm{H} \beta$ line, we analysed the continuum luminosity, $L_{\lambda}(5100 \AA)$, derived from the incident SED (Figure 1), and checked what radius of the $\mathrm{H} \beta$ emission we should expect from observational measurements (Kilerci Eser et al. 2015). For $\log L_{\lambda}(5100 \AA) \approx 43.25$ used in our model, we have a delay in $\mathrm{H} \beta$ of the order of 20 days and line width FWHM $\approx 4700 \mathrm{~km} \mathrm{~s}^{-1}$. Similar results were obtained by Peterson et al. (1991) using ground-based observations made in 1989 , where a delay of 21 days between continuum and $\mathrm{H} \beta$ was reported. Those observed parameters correspond to the radius at which the $\mathrm{H} \beta$ line luminosity reaches its maximum, about $4 \times 10^{17} \mathrm{~cm}$ in our model (Figure 11 third panel).

However, recent observations of velocity-resolved reverberation mapping presented by Pei et al. (2017) show new points that do not agree with the fitted trend of $L_{\lambda}(5100 \AA)$ versus $\mathrm{H} \beta$ delay (Kilerci Eser et al. 2015). Those new points for $\log L_{\lambda}(5100 \AA) \gtrsim 43.3$ present an $\mathrm{H} \beta$ delay of the order of 3 days, so shorter than even for the state of lowest luminosity, $\log L_{\lambda}(5100 \AA) \approx 42.5$. Lu et al. (2016) derived the delay in BLR response to the change in source luminosity to be $2.4 \mathrm{yr}$. During this time, the BLR may be rebuilt under a change of radiation pressure, thus making the comparison of our model to the data more difficult.

The emissivity profile of both LILs $\mathrm{H} \beta$ and Mg II in NGC 5548 is very similar in our model (Figure 12 third panel). However, this is not exactly the case in reverberation mapping observations. The $\mathrm{Mg}$ II line is more puzzling in this case. Clavel et al. (1991) presented peak-center delays from a multimonth IUE campaign undertaken in 1989. They found a very broad response in the $\mathrm{Mg}$ II line covering 34-72 days. In addition, Cackett et al. (2015) analyzed Mg II variability and
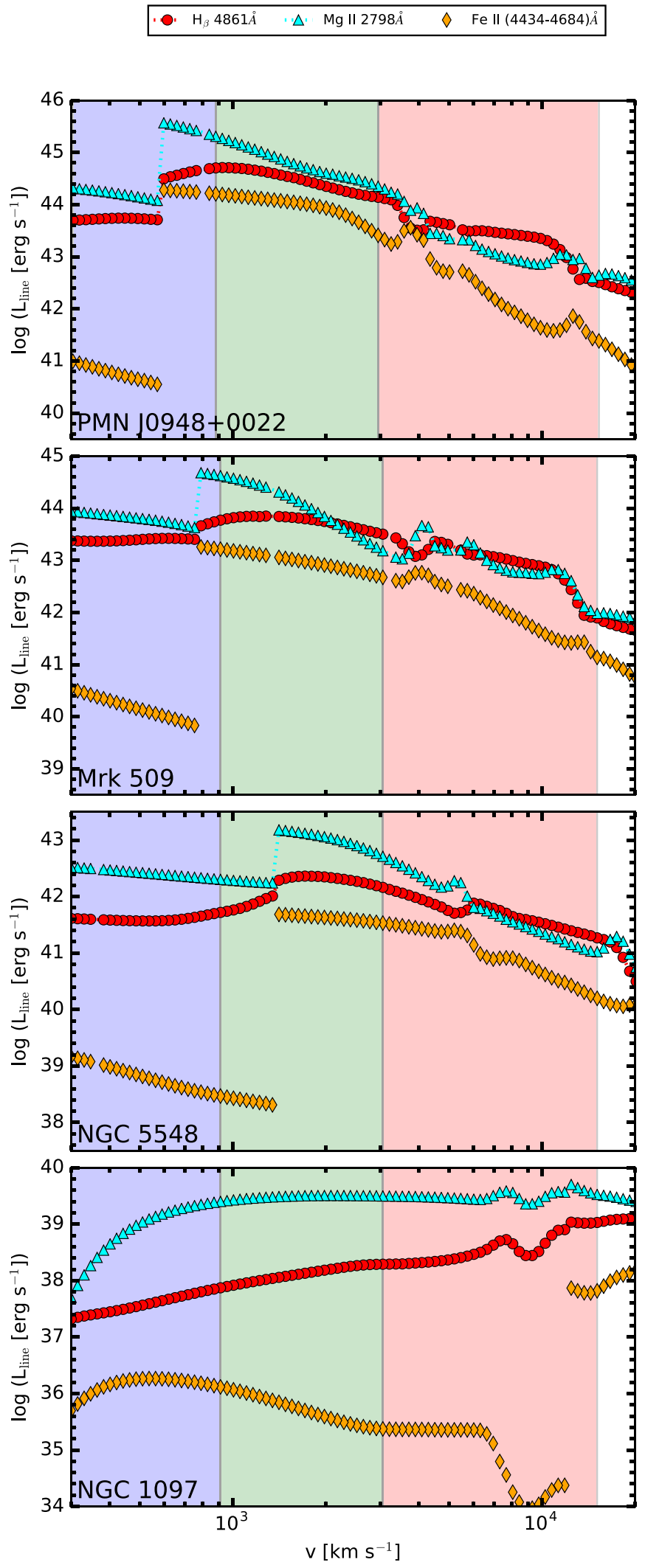

Figure 12. Luminosities of LILs vs. Keplerian velocity obtained for disk-like cloud density profiles. Each panel shows one type of AGN such that the source luminosity (given in Table 1) decreases from top to bottom. Shaded areas mark the positions of the BLR, ILR, and NLR from right to left, based on the adopted range of Keplerian velocities (see the text for details). 


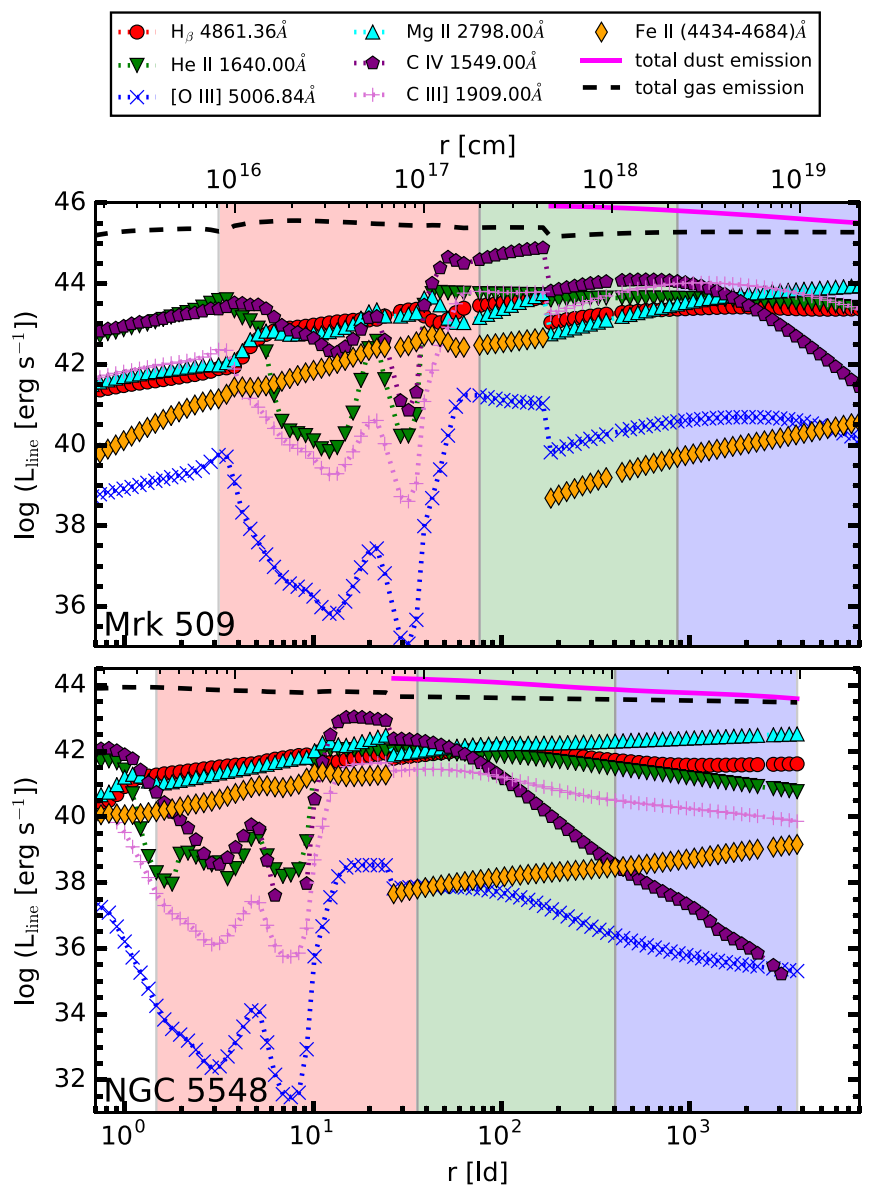

Figure 13. The same as in Figure 11 for the two best observed sources, Mrk 509 (upper panel) and MGC 5548 (lower panel), but for a sublimation radius computed from the formula $R_{\mathrm{d}}=0.06 \sqrt{L / 10^{45}}$ (Laor \& Draine 1993).

found only a weakly correlated broad response to the continuum brightening, with the delay in response in the range 20-70 days. Both results may suggest that the global maximum of line luminosity is located in the ILR, which fully agrees with our model. However, an even shallower global maximum of line luminosity in $\mathrm{H} \beta$ located in the same region of our model is not resolved in the observations of delay. $\mathrm{Mg}$ II shows a more luminous ILR with maximum before the face of the torus. A brighter ILR in the magnesium line should be reflected in higher average delays than in hydrogen Balmer lines, and this is the case in reverberation measurements.

For the case of NGC 5548, Clavel et al. (1991) presented light curves of $\mathrm{C}$ III] and $\mathrm{C}$ IV for which the delay covers approximately 26-32 days and 8-16 days respectively. This is consistent with the position of the global maximum in the emissivities of those lines further on and at the outer edge of the dense BLR, which fully agrees with our model. This is also explained by Negrete et al. (2013), who inferred the emission radius of those HILs from the photoionization condition. They found optimal emission of C IV for $n_{\mathrm{H}}=10^{12} \mathrm{~cm}^{-3}$ and $\log U \approx-2$, and of $\mathrm{C}$ III] for $n_{\mathrm{H}}=10^{10} \mathrm{~cm}^{-3}$ and $\log U \approx$ -1.5 , which is consistent with our model as seen in Figure 10. C III] emissivity in the ILR is rather flat and allows noticeable emission to originate from clouds located at higher radii.

The permitted He II line is always broad and blended with semi-forbidden $\mathrm{O} \mathrm{III}]$, therefore the measurements of delay covering 4-10 days are more difficult to explain with our model. Such a delay corresponds to the location of the drop in emissivity of the He II line in the dense BLR in NGC 5548 (see third panel of Figure 11). Our model predicts that the local maximum in He II luminosity is located on the outer edge of the dense BLR for the expected delay of $\approx 20$ days.

HST monitoring described by De Rosa et al. (2015) reveals a $\mathrm{C}$ IV delay of 5 days and He II delay of 2.5 days. This again corresponds to the drop in emissivity of our model. Those results may demand stronger modification of our model. For instance, gaseous clouds present at a higher elevation above the disk where the density departs from the atmospheric value may be significant. Such a geometry for broad HIL regions has already been postulated in the literature (i.e., Collin et al. 2006; Decarli et al. 2008; Kollatschny \& Zetzl 2013).

To check how the position of the dusty torus (discussed in Section 4) influences the radial emissivity profile, we present in Figure 13 the case of the two best studied sources computed for a 10 times smaller value of sublimation radius taken as $R_{\mathrm{d}}=$ $0.06 \sqrt{L / 10^{45}}$ (Laor \& Draine 1993). For Mrk 509, Koshida et al. (2014) have reported a delay of the dust phase by 120-150 days, depending on the method of derivation. This value fully agrees with the position of the sublimation radius from the formula by Laor \& Draine (1993), presented in the upper panel of Figure 13. However, this fact would eliminate the dominance of the $1000 \mathrm{~km} \mathrm{~s}^{-1}$ component in the $\mathrm{Mg}$ II line and possibly would remove the intermediate-width line component of the global emissivity maximum of $\mathrm{H} \beta$. This brings our model closer to the observed line delays. In the case of NGC 5548, Koshida et al. (2014) derived the radius of the face of the inner torus by comparing optical and near-infrared variability. Their measurements range from 60 to 80 days depending on the continuum luminosity of NGC 5548 and the method used in calculations. This is over three times larger than the sublimation radius found by Laor \& Draine (1993) (lower panel of Figure 13), and half that computed by Equation (14). The lower position of the sublimation radius in NGC 5548 influences the maximum emissivity of $\mathrm{Mg}$ II, shifting it to lower radii and decreasing the contribution from the $2000 \mathrm{~km} \mathrm{~s}^{-1}$ component.

Peterson et al. (2013) investigated the variability of the forbidden [O III] line in NGC 5548. They found a delay of $10-20 \mathrm{yr}(\sim 2-3 \mathrm{pc})$ and suggested an emitting medium with a density of $10^{5} \mathrm{~cm}^{-3}$. The NLR studied in X-rays, as suggested by Detmers et al. (2009), covers $1-15 \mathrm{pc}$, or more precisely $14 \mathrm{pc}$ as derived by Whewell et al. (2015). In our model, densities corresponding to the NLR remain high $\left(\approx 10^{9} \mathrm{~cm}^{-3}\right)$. We have high emissivities of narrow components in all permitted lines except $\mathrm{C}$ IV. In addition, [O III] emissivity remains rather low. Crenshaw et al. (1993) reported strong narrow components in all optical/UV permitted lines, especially C IV. Thus our model is less accurate at reproducing the NLR for an assumed disk-like density profile.

The model of Mrk 509 is in many respects similar to that of NGC 5548. The most noticeable difference is a shift in the maximum emissivity of C III] and C IV toward greater radii. This predicts stronger intermediate emission line components than our model. And this seems to be the case when we look at the observational spectra, presented for example by Negrete et al. (2013). Our model computed for the NLSy1 galaxy PMN J0948 shows very similar shapes of emissivity profile to those of Mrk 509 because those sources have similar BH masses but different SEDs. However, the ratio of line luminosity to 
continuum luminosity is lower for NLSy1, thus broad components effectively blend with the continuum and only the contrast of narrow components remains sufficient to make the line visible. The LINER case of NGC 1097 is exceptional because the sublimation radius is inside our dense BLR. This fact makes all line luminosity profiles flat up to the NLR, which is in agreement with Balmaverde et al. (2016) who emphasized the extended ILR in LINERS up to $10 \mathrm{pc}$. In the case of a flat radial luminosity profile, the narrow component has the highest contrast and therefore it will dominate the line profile, which is in agreement with González-Martín et al. (2015), who pointed out that AGNdominated LINERs are very similar to Seyfert 2 galaxies.

\section{Conclusions}

We carried out photoionization simulations of the ionized gas clouds in AGNs and studied the effect of varying density profiles on the line emission across the radial distance that spans all the way from the BLR down to the NLR. The different density prescriptions are applied in the following ways: (a) we employed the density profile as a simple power law used by NL93 and AD16, and varied its slope; (b) we self-consistently computed disk-like density profiles for each AGN by using their observed properties, i.e., black hole mass and Eddington ratio.

Using the various density profiles derived, we computed the line luminosities of the major emission lines in Sy1.5 Mrk 509, Sy1 NGC 5548, NLSy1 PMN J0948+0022, and LINER NGC 1097, which differ in their SEDs. Below we list final conclusions.

1. In the case of clouds located at distances considered in this paper, $\mathrm{CP}$ and $\mathrm{CD}$ cloud models reproduce exactly the same line luminosity profiles in the regime of lines observed in the optical/UV. This is caused by the fact that our clouds are dense. Less dense clouds do not produce the ILR.

2. The varying slope of the power-law density profile does not affect the nature of the ILR. In particular, the intermediate emission in $\mathrm{H} \beta$ is present for all the slopes independent of the SED shape.

3. For the lower slope of the density profile, forbidden [O III] and semi-forbidden $\mathrm{CIII}$ ] lines are strongly suppressed because of the high-density environment. As the slope becomes steeper, i.e., density decreases, these lines are prominent at radial distances corresponding to the NLR.

4. The Fe II emission line appeared to be most sensitive to the presence of dust, since its luminosity drops by two orders of magnitude at the sublimation radius (see Section 3 for details).

5. The drop in density in the disk-like density profiles causes mild enhancement of $\mathrm{Mg}$ II, $\mathrm{H} \beta$, and Fe II lines, while He II, C III], and [O III] are suppressed at the location of this drop. This result is consistent with separation of LIL and HIL clouds in a two-component BLR model (Collin-Souffrin et al. 1988).

6. The low luminosity of the LINER NGC 1097 shifts the dust sublimation radius toward smaller distances from the $\mathrm{SMBH}$, which makes the emissivity profiles of all lines flat. Therefore, an intermediate-line component can be detectable, but it is less prominent than the narrow-line component.

7. The distance inferred from the time delay of $\mathrm{H} \beta$ and Mg II in NGC 5548 taken from reverberation mapping closely agrees with the distance at which the $\mathrm{H} \beta$ line peaks in the simulated line emissivity profile.
8. The NLR from our disk-like model is denser than is postulated from observations. NLR clouds may become rare while escaping from the accretion disk atmosphere, which we plan to take into account in a future paper.

This research was supported by Polish National Science Center grants No. 2015/17/B/ST9/03422, 2015/18/M/ST9/ 00541, 2015/17/B/ST9/03436, 2016/21/N/ST9/03311 and by Ministry of Science and Higher Education grant W30/7.PR/ 2013. It received funding from the European Union Seventh Framework Program (FP7/2007-2013) under the grant agreement No.312789. T.P.A. received funding from NCAC PAS grant for young researchers. G.J.F. thanks the Nicolaus Copernicus Astronomical Center for its hospitality and acknowledges support by NSF (1108928, 1109061, and 1412155), NASA (10-ATP10-0053, 10-ADAP10-0073, NNX12AH73G, and ATP13-0153), and STScI (HST-AR-13245, GO-12560, HST-GO-12309, GO-13310.002-A, HST-AR-13914, and HSTAR-14286.001).

Software: CLOUDY (v17.00; Ferland et al. 2017).

\section{ORCID iDs}

T. P. Adhikari (iD https://orcid.org/0000-0003-4586-0744

B. Czerny (iD https://orcid.org/0000-0001-5848-4333

G. J. Ferland (iD https://orcid.org/0000-0003-4503-6333

\section{References}

Abdo, A. A., Ackermann, M., Ajello, M., et al. 2009, ApJ, 699, 976 Adhikari, T. P., Różańska, A., Czerny, B., Hryniewicz, K., \& Ferland, G. J. 2016, ApJ, 831, 68

Adhikari, T. P., Różańska, A., Hryniewicz, K., Czerny, B., \& Ferland, G. J. 2017, FrASS, 4, 19

Adhikari, T. P., Różańska, A., Sobolewska, M., \& Czerny, B. 2015, ApJ, 815,83

Balmaverde, B., Capetti, A., Moisio, D., Baldi, R. D., \& Marconi, A. 2016, A\&A, 586, A48

Baskin, A., \& Laor, A. 2018, MNRAS, 474, 1970

Baskin, A., Laor, A., \& Stern, J. 2014, MNRAS, 438, 604

Bentz, M. C., Denny, K. D., Cackett, E. M., et al. 2007, ApJ, 662, 205

Bentz, M. C., Peterson, B. M., Pogge, R. W., Vestergaard, M., \& Onken, C. A. 2006, ApJ, 644, 133

Boissay, R., Ponti, G., Bianchi, S., et al. 2014, A\&A, 567, A44

Boroson, T. A., \& Green, R. F. 1992, ApJS, 80, 109

Brotherton, M. S., Wills, B. J., Francis, P. J., \& Steidel, C. C. 1994, ApJ, 430, 495

Cackett, E. M., Gültekin, K., Bentz, M. C., et al. 2015, ApJ, 810, 86

Clavel, J., Reichert, G. A., Alloin, D., et al. 1991, ApJ, 366, 64

Collin, S., Kawaguchi, T., Peterson, B. M., \& Vestergaard, M. 2006, A\&A, 456, 75

Collin-Souffrin, S., Dyson, J. E., McDowell, J. C., \& Perry, J. J. 1988, MNRAS, 232, 539

Crenshaw, D. M., Boggess, A., \& Wu, C.-C. 1993, ApJL, 416, L67

Crenshaw, D. M., \& Kraemer, S. B. 2007, ApJ, 659, 250

Crenshaw, D. M., Kraemer, S. B., Schmitt, H. R., et al. 2009, ApJ, 698, 281

Czerny, B., \& Hryniewicz, K. 2011, A\&A, 525, L8

Czerny, B., Li, J., Loska, Z., \& Szczerba, R. 2004, MNRAS, 348, L54

Czerny, B., Li, Y. R., Hryniewicz, K., et al. 2017, ApJ, 846, 154

Czerny, B., Modzelewska, J., Petrogalli, F., et al. 2015, AdSpR, 55, 1806

D’Ammando, F., Orienti, M., Finke, J., et al. 2015, MNRAS, 446, 2456

Davidson, K. 1972, ApJ, 171, 213

Decarli, R., Labita, M., Treves, A., \& Falomo, R. 2008, MNRAS, 387, 1237

Denney, K. D., Peterson, B. M., Pogge, R. W., et al. 2009, ApJL, 704, L80

De Rosa, G., Peterson, B. M., Ely, J., et al. 2015, ApJ, 806, 128

Detmers, R. G., Kaastra, J. S., \& McHardy, I. M. 2009, A\&A, 504, 409

Dopita, M. A., Groves, B. A., Sutherland, R. S., Binette, L., \& Cecil, G. 2002, ApJ, 572, 753

Dumont, A.-M., Abrassart, A., \& Collin, S. 2000, A\&A, 357, 823

Elvis, M. 2004, in ASP Conf. Ser. 311, AGN Physics with the Sloan Digital Sky Survey, ed. G. T. Richards \& P. B. Hall (San Francisco, CA: ASP), 109 
Ferland, G. J., Chatzikos, M., Guzman, F., et al. 2017, RMxAA, 53, 385 Foschini, L., Ghisellini, G., Kovalev, Y. Y., et al. 2011, MNRAS, 413, 1671 Gaskell, C. M. 2009, NewAR, 53, 140

Gaskell, C. M. 2017, MNRAS, 467, 226

Goad, M. R., \& Korista, K. T. 2014, MNRAS, 444, 43

Goad, M. R., Korista, K. T., \& Ruff, A. J. 2012, MNRAS, 426, 3086

González-Martín, O., Masegosa, J., Marquez, I., et al. 2015, A\&A, 578, A74

Grevesse, N., \& Sauval, A. J. 1998, SSRv, 85, 161

Ho, L. C., \& Kim, M. 2014, ApJ, 789, 17

Hu, C., Wang, J.-M., Ho, L. C., et al. 2008a, ApJL, 683, L115

Hu, C., Wang, J.-M., Ho, L. C., et al. 2008b, ApJ, 687, 78

Kaastra, J. S., Petrucci, P. O., Cappi, M., et al. 2011, A\&A, 534, A36

Kilerci Eser, E., Vestergaard, M., Peterson, B. M., Denney, K. D., \& Bentz, M. C. 2015, ApJ, 801, 8

Kollatschny, W., \& Zetzl, M. 2013, A\&A, 551, L6

Koshida, S., Minezaki, T., Yoshii, Y., et al. 2014, ApJ, 788, 159

Krolik, J. H., McKee, C. F., \& Tarter, C. B. 1981, ApJ, 249, 422

Laor, A., \& Draine, B. T. 1993, ApJ, 402, 441

Lu, K.-X., Du, P., Hu, C., et al. 2016, ApJ, 827, 118

Lyu, J., Hao, L., \& Li, A. 2014, ApJL, 792, L9

Maiolino, R., Marconi, A., Salvati, M., et al. 2001, A\&A, 365, 28

Mason, K. O., Puchnarewicz, E. M., \& Jones, L. R. 1996, MNRAS, 283, L26

Mehdipour, M., Branduardi-Raymont, G., Kaastra, J. S., et al. 2011, A\&A, 534, A39
Mehdipour, M., Kaastra, J. S., Kriss, G. A., et al. 2015, A\&A, 575, A22

Negrete, C. A., Dultzin, D., Marziani, P., \& Sulentic, J. W. 2013, ApJ, 771, 31

Nemmen, R. S., Storchi-Bergmann, T., \& Eracleous, M. 2014, MNRAS, 438, 2804

Nenkova, M., Sirocky, M. M., Ivezić, Ž, \& Elitzur, M. 2008, ApJ, 685, 147

Netzer, H. 1990, Active Galactic Nuclei, ed. R. D. Blandford et al., 57

Netzer, H., \& Laor, A. 1993, ApJL, 404, L51

Onishi, K., Iguchi, S., Sheth, K., \& Kohno, K. 2015, ApJ, 806, 39

Osterbrock, D. E., \& Ferland, G. J. 2006, Astrophysics of Gaseous Nebulae and Active Galactic Nuclei (Sausalito, CA: University Science Books)

Pei, L., et al. 2017, ApJ, 837, 131

Peterson, B. M., Balonek, T. J., Barker, E. S., et al. 1991, ApJ, 368, 119

Peterson, B. M., Denney, K. D., De Rosa, G., et al. 2013, ApJ, 779, 109

Peterson, B. M., Ferrarese, K., Gilbert, K. M., et al. 2004, ApJ, 613, 682

Różańska, A., Czerny, B., Życki, P. T., \& Pojmański, G. 1999, MNRAS, 305,481

Różańska, A., Goosmann, R., Dumont, A.-M., \& Czerny, B. 2006, A\&A, 452,1

Różańska, A., Kowalska, I., \& Gonçalves, A. C. 2008, A\&A, 487, 895

Shakura, N. I., \& Sunyaev, R. A. 1973, A\&A, 24, 337

Whewell, M., Branduardi-Raymont, G., Kaastra, J. S., et al. 2015, A\&A, 581, A79

Xie, Y., Li, A., \& Hao, L. 2017, ApJS, 228, 6

Zhu, L., Zhang, S. N., \& Tang, S. 2009, ApJ, 700, 1173 